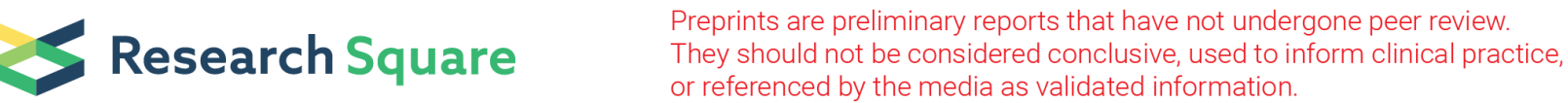

\section{Diet Induces the Difference of Gut Fungal Communities between Anser erythropus Wintering at Shengjin and Caizi Lakes, China}

Gang Liu ( $D$ liugang8966@163.com )

Anhui Medical University

Zhizhong Gong

Anhui Medical University

Jiahui Feng

Anhui Medical University

$\mathrm{Na} \mathrm{Xu}$

Anhui Medical University

Research Article

Keywords: Anser erythropus, Gut fungal community, Pathogen, OUT, alpha diversity

Posted Date: August 13th, 2021

DOl: https://doi.org/10.21203/rs.3.rs-795606/v1

License: (c) (i) This work is licensed under a Creative Commons Attribution 4.0 International License.

Read Full License 


\section{Abstract}

The gut microbiota play major roles in host nutrition and metabolism, and even potential to cause serious disease for animals and human, however, the knowledge of waterbirds' gut fungal communities are quite limited at present. In this paper, the gut fungal communities and infer the potential pathogens isolated from the feces of Anser erythropus wintering at Shengjin Lake (SJ) and Caizi Lake (CZ) were investigated based on ITS gene region by using high-throughput sequencing. 1,302,562 valid tags corresponding to 2,102 OTUs were retained from $20 \mathrm{fecal}$ samples, including 10 samples per lake. The OTUs from SJ geese represented seven phyla and 27 classes, seven phyla and 28 classes were identified from $\mathrm{CZ}$ samples. Ascomycota, Basidiomycota, Zygomycota and Rozellomycota were the dominant gut fungal phyla in this study, accounted for $61.60 \%, 35.60 \%, 1.84 \%$, and $0.30 \%$ of the OTUs, respectively. The alpha diversity indices showed significantly different between the geese from SJ and CZ. The Anser erythropus mainly ate Poaceae spp. at SJ, while Carex spp. component was identified at CZ, suggesting that the variations in fungal community between the two lake geese might be induced by different diets. We also observed a fungal pattern with a higher number of significant correlations to bacterial genus, and Ceratobasidium, Tomentella, Paurocotylis, Tuber, Podospora and Mortierella were core fungal genus in the two lake geese. Nine potential pathogenic species were identified in the guts across all samples of Anser erythropus at $\mathrm{SJ}$ and $\mathrm{CZ}$, it also showed the relative abundance of potential pathogen was significantly higher from SJ samples than that from CZ samples. These findings expanded our knowledge on the gut fungi for waterbirds, indicating the fungi are highly sensitive to diet at two lakes and should pay more attention to the potential pathogenic species of Anser erythropus.

\section{Introduction}

Gut microbiota ecosystem in animals and human is complex and plays great importance for acting the role of gastrointestinal system protection and metabolism, eliminating toxic metabolites and drugs, protecting and regulating immune system responses of host to various pathogens, and helping with the development and maturation of immune cells [1-4]. More and more recent studies have highlighted the importance of gut microbial communities are increasingly appreciated, however, the processes that govern these communities are far from clear [1-4]. Previous studies have been indicated that host was a major factor influencing the gut microbial community structure and function, and diets, ages, habitat and behavior also might influence their composition and structure [5-9]. Habitat and diet were considered to be the main factors contributing to gut microbial abundance, diversity, structure and function $[4,10]$. Environmental factors also alter the composition of gut microflora, and it is considered that gut microbiota may be significantly differ among members of the same species from different habitats or geographical areas $[4,10]$. The fungal population only makes up at most $0.1 \%$ of the microorganisms in the gut, and the role of them has been ignored in the past decades, however, recently several studies indicated that fungi might be contribute to the metabolism and intestinal health, include provisioning of essential amino acids, contributing to lignocellulose degradation and increasing nutrition levels for their hosts $[4,10-11]$. However, due to the relatively lower abundance and lack of well-characterised reference 
genome data, fungi are largely unexplored in animal [11-13]. Nevertheless, among Aves, gut fungi research has mainly focused on poultry and pet birds, as a result, the knowledge of their contributions to birds is limited. Thus, understanding gut fungi of wildbirds might be important for clarifying their function for the hosts.

Birds are interesting study groups in which to investigate the roles of gut microbes, because they have extremely complex and unique diets, physiological traits, and developmental strategies [14]. Because of their flying ability and unique life history such as migratory behavior, complex dietary and nest habitats, additionally, they lives at various habitats during migration, which can get and spread microbes over vast distances, representing an interesting and best-studied groups for gut microbiota [14]. As high-throughput sequencing development, the knowledge of gut microbiome about migratory waterbirds has markedly increased during past few years [15-16]. Anser erythropus is known as lesser white-fronted goose, is a long-distance migratory waterbird and an important indicator species in the wetland. The species belongs to Anatidae, Anseriformes, mainly breeds from the northeastern Siberia to Fennoscandian Lapland, and mainly winter in China, South Korea and Japan. In China, these geese mainly winter at SJ and CZ along the middle and lower Yangtze River floodplains from October to next April [17]. Gut bacterial communities has been shown that dramatic shifts between Anser erythropus wintering at SJ and CZ [10]. However, little is known about the gut fungal community and function of this species, especially the knowledge of them wintering at SJ and CZ. Thus, understanding the gut fungal communities about Anser erythropus might be important for this species to clarify their ecological structure and function.

Shengjin and Caizi Lakes $\left(30.25^{\circ}-30.50^{\circ} \mathrm{N}, 116.92^{\circ}-117.25^{\circ} \mathrm{E} ; 30.75^{\circ}-30.97^{\circ} \mathrm{N}, 117.00^{\circ}-117.15^{\circ} \mathrm{E}\right.$, respectively) are internationally important wetlands, both of them are shallow and river-connected lake in the middle of the Yangtze River. Both of the two lakes contain abundant aquatic resources for migratory watebirds, they are important stopover and wintering wetlands in East Asian-Australasian fly for migratory waterbirds $[10,18]$. In this study, we want to investigate and analyze the gut fungal community composition, structure and fuction of Anser erythropus wintering at SJ and CZ by using high-throughput sequencing of the ITS region, and we also want to answers for the following questions: (a) Are taxonomical structures of fungal communities of SJ different from those of CZ? (b) What are key factor that affect the structure of fungal communities could differentiate samples?

\section{Materials And Methods}

\section{Sample Collection}

20 fecal samples including ten fecal samples wintering at SJ and ten fecal samples from Anser erythropus wintering at $\mathrm{CZ}$ were collected on December in paddy lands. The sample collection methods were referred to Dong and Xiang, noninvasive techniques were used and no birds were harmed in this study [19-20], this research was approved by the Animal Research Ethics Committee of Anhui Medical University. Before the fecal samples were collected, large groups containing more than 150 geese was select by used binoculars to observe. The distance was more than 3-5 m among goose fecal samples to 
avoid individual repetition. All fecal samples were placed into sterile $50 \mathrm{ml}$ centrifuge tubes and transported to the laboratory immediately, then stored at $-80^{\circ} \mathrm{C}$.

\section{Fecal DNA Extraction, Species Determination and High-throughput Sequencing}

Fecal sample DNA was isolated using a MagPure Soil DNA LQ Kit (Magen, Guangdong, China). Avian species determination was used COI gene with primer BIRDF1/BIRDR1, the detailed information about DNA extraction, PCR reaction and sequences identified were referred to Xiang [20], and make sure all fecal samples were confirmed to be Anser erythropus. PCR amplification of the ITS region was carried out in a $25 \mu$ reaction using universal primer pairs (ITS1F-5'-CTT GGT CAT TTA GAG GAA GTA A-3'; ITS2-5'-GCT GCG TTC TTC ATC GAT GC-3'). The PCR products were purified with Agencourt AMPure XP beads (Beckman Coulter Co., USA) and Qubit dsDNA assay kit were used for quantifying, and the concentrations were then adjusted for sequencing. An Illumina NovaSeq 6000 with two paired-end read cycles of 250 bases each was used for performing sequence platform (Illumina Inc., San Diego, CA; OE Biotech Company, Shanghai, China). The data of raw reads has been submitted to the SRA (Sequence Read Archive) of the NCBI (accession numbers SRR15328848).

\section{Bioinformatics and Statistical Analysis}

The fungal data of raw sequencing were in FASTQ format and processed by QIIME (v2-2020.2). Trimmomatic software was used to preprocess the paired-end reads to filter low quality sequences. Then, reads with chimera were filtered using VSEARCH. Clean reads were subjected to primer sequences removal and clustering to generate operational taxonomic units (OTUs) using VSEARCH software with $97 \%$ similarity cutoff. All representative reads were annotated and blasted against Silva database (Version 132) using RDP classifier (confidence threshold was 70\%). The gut fugal diversity of Anser erythropus was estimated using the alpha diversity including Chao1, Shannon and Simpson indexes by One-way ANOVA method. The Unifrac distance matrix performed by QIIME software was used for unweighted Unifrac Principal coordinates analysis (PCoA).

\section{Results}

\section{General Sequencing Information}

The ITS gene region of 20 fecal samples collected from wintering Anser erythropus at SJ and CZ was sequenced and analyzed. After removing low-quality reads, 1,302,562 valid tags corresponding to 2,102 OTUs were retained. An average of 147 OTUs (range, 57-774 per sample) was contained in each sample, $96.5 \%$ (average) were classified as fungal phyla. Of all the OTUs, $44.09 \%(887 / 2,102)$ was found in both lake geese (Figure 1A). Geese from SJ had $41.58 \%$ unique OTUs $(874 / 2,102)$, and $14.33 \%(341 / 2,102)$ unique OTUs was found in CZ geese (Figure 1A), and a total of five core-OTUs were identified in this study (Figure 1B).

\section{Alpha and Beta Diversity}


Gut fungal alpha diversity in gut fungi from Anser erythropus was estimated via the observed Chao1, Simpson and Shannon indices. The Chao1, Simpson, and Shannon indices indicated differed significantly between the SJ and CZ geese (Figure 2). One-way ANOVA results showed that alpha diversity in the gut for the SJ geese was significantly higher than that for the $\mathrm{CZ}$ geese $(p<0.05)$. Fungal beta diversity was further assessed by Unweighted Pair Group Method with Arithmetic Mean (UPGMA) clustering using the unweighted UniFrac distance matrix. PCoA analysis result indicated that the gut fungal community was significantly difference between the guts of the geese from $\mathrm{SJ}$ and $\mathrm{CZ}$, and most samples were well matched with their sampling lakes (Figure 3).

\section{Gut Fungal Community Composition}

The gut fungi of fecal samples from Anser erythropus at SJ contained seven phyla and 27 classes, whereas seven phyla and 28 classes were identified in the $C Z$ sample. The dominant phyla of gut fungi from two lake geese were Ascomycota, Basidiomycota, Zygomycota, Rozellomycota, Glomeromycota, Chytridiomycota, Cercozoa. Ascomycota, Basidiomycota, Zygomycota and Rozellomycota accounted for $61.60 \%, 35.60 \%, 1.84 \%$, and $0.30 \%$ of the OTUs, respectively (Figure 4). Glomeromycota, Chytridiomycota and Cercozoa accounted for less than $0.001 \%$ of all. The fecal samples of geese collected from SJ had significantly higher relative abundance of Basidiomycota, Zygomycota, Rozellomycota, Glomeromycota than that collected from $\mathrm{CZ}$, however, the relative abundance of Ascomycota, Chytridiomycota, Cercozoa indicated that the species diversity was no significant difference between two samples.

LEfSe analysis result showed differences in the abundances of specific gut fungal taxa from the Anser erythropus at the two lakes. Basidiomycota was significantly much more abundant in geese from CZ, whereas Nectriaceae and Sordariales were significantly much more abundant in the samples from SJ (Figure 5). Spearman's rank correlation test was employed to identify the co-occurrence patterns among the top 30 core genera (Figure 6). Zopfiella and Staphylotrichum had a very strong co-exclusion relationship $(\mathrm{R}=-0.982, p<0.001)$, however, Tomentella only had a very weak co-exclusion relationship with Staphylotrichum $(\mathrm{R}=-0.270, p=0.253)$ (Figure 6).

\section{Bacteria-fungi Association Analysis}

We analyzed whether the fungal communities was correlated with the bacterial communities, the gut bacterial communities date of 16S rRNA V3-V4 region sequences for the same 20 fecal sample date out of 30 samples from SJ and CZ used our previous study data [10]. The results showed some fungal genus were significant correlations to bacterial genus in the 20 geese fecal samples (Figure 7). Interestingly, there was a positive correlation between the abundance of bacterial genus (Sphingobacterium, Brevundimonas, Stenotrophomonas, Chryseobacterium, Acinetobacter,Pseudomonas) and the abundance of several fungal genus (Tuber, Paurocotylis, Mortierella, Archaeorhizomyces, Acremonium, Subulicystidium, Podospora, Ceratobasidium, Tomentella, Erythrobasidium, Cystofilobasidium), and Sporosarcina and Lactobacillus was a negative correlation with Paurocotylis, Mortierella, Archaeorhizomyces, Acremonium, Subulicystidium, Podospora, Ceratobasidium, Tomentella (Figure 7). Bivariate comparisons between Shengjin and Caizi Lakes revealed significant Pearson correlation 
coefficients for fungi community and bacterial community $(R=-0.52)$. Correlation networks was also built at the genus level involving both bacteria and fungi, it showed Sphingobacterium, Stenotrophomonas, Pseudomonas, Brevundimonas and Chryseobacterium are core bacterial genus, and Ceratobasidium, Tomentella, Paurocotylis, Tuber, Podospora and Mortierella are core fungal genus (Figure 8).

\section{Potential Pathogen}

We identified nine potential pathogenic species in the guts across all samples of Anser erythropus in this study. The results showed that the relative abundance of potential fungal pathogen was significantly higher from SJ geese than that from CZ samples (Figure 9A-B). Basidiomycota (Malassezia restricta, Cryptococcus heimaeyensis, Papiliotrema laurentii, Apiotrichum veenhuisii) and Ascomycota (Graphium basitruncatum, Purpureocillium avendulum, Aspergillus flavus, Monocillium indicum, Hirsutella vermicola) were the dominant potential pathogenic phylum in guts of geese at both two lakes. Malassezia restricta may cause Crohn's disease, Cryptococcus heimaeyensis may cause leukemia, and Papiliotrema laurentii may cause immune system disease in humans. Previous study reported that Apiotrichum veenhuisii was isolated from the skin of a pediatric patient with acute myeloid leukemia [21]. Graphium basitruncatum, Purpureocillium avendulum, Aspergillus flavus, Monocillium indicum and Hirsutella vermicola may widely propagate their gut pathogens and increase the risk of disease in other animals, even human beings. However, samples of SJ6, CZ1 and CZ5 hadn't identified any potential pathogenic species.

\section{Discussion}

Migratory waterbirds travel long distances in life history, they also utilize diverse habitats and found in various ecosystems during migration process, resulting in complex and diverse gut fungi [20]. Recent study indicated the gut of Anser erythropus contain a large number of bacteria and spread them over vast distance, it also showed dramatic shifts between CZ and SJ geese [10]. As another important group of the gut microbial ecosystem, fungi play a critical role in the metabolism and health of their host [22]. However, people pay little attention to the feature of gut fungi in waterbirds, this study is the first to report the gut fungi from Anser erythropus wintering along the middle and lower reaches of the Yangtze River in eastern China.

The gut fungal community of the Anser erythropus was dominated by Ascomycota (61.60\%), Basidiomycota (35.60\%), Zygomycota (1.84\%) and Rozellomycota (0.30\%), which was similar to Grus monacha winter at SJ [14]. Ascomycota and Basidiomycota were dominated maybe associated with these geese mainly eat the roots and leaves of plants during wintering period at two lakes, these fungi could help birds to digest and absorb higher abundance of plant saprotroph $[14,17,22]$. Tomentella may be associated with the produce of acetate in the gut of birds, suggesting that the gut fungi might be involved in the metabolism of dietary polysaccharides [14]. Archaeorhizomyces and Ceratobasidium might increase host's digestion and immunity. Tomentella, Archaeorhizomyces and Ceratobasidium were dominated in Anser erythropus gut at the two lakes, which are different with Grus monacha at SJ [14]. All 
the results suggested that the Anser erythropus might depend more on their gut fungi to acquire nutrients and enhance immunity, which are different with Grus monacha at SJ [14].

Studies have indicated that the composition and function of gut bacteria in waterbirds was deeply influenced by environment, such as diet, lifestyle and seasonal fluctuations $[11,14,23]$. The alpha diversity in the gut fungal community was significant difference between Anser erythropus wintering at SJ and CZ in this study, which is similar to gut bacteria community [10]. However, relative abundance were significantly higher in SJ samples than that in CZ samples, which is difference from gut bacteria community results, support that the gut fungal communities were strongly associated with the gut environment filtering [10]. SJ and CZ provide abundant and diverse food sources with Anser erythropus while wintering, however, the diets were large differences of the geese at the two lakes, at SJ, the Anser erythropus mainly ate Poaceae spp, while Carex spp component was identified at CZ $[17,22,24]$. Thus, the dramatic variations in gut fungal communities might be induced by different diets between the two lakes. In addition, the Anser erythropus winter at Shengjin Lake provide had higher gut fungal diversity than geese winter at Caizi Lake, the effect of environmental changes on the wintering Anser erythropus relating to fugal alpha diversity was consistent between $\mathrm{SJ}$ and $\mathrm{CZ}$, which is similar to gut microbiota community results [10].

Birds are an important source of disease source and vector in environment, they can spread many diseases through water, soil or air contamination to poultry and even human [3,25]. As a kind of migratory birds, Anser erythropus fly long distance and live at various habitats in their life history, thus, they may carry and spread a great many pathogens. Nine potential pathogens were identified in this study, they may cause serious disease to humans and/or other animals. In this study, Anser erythropus from SJ carried much more gut potential pathogenic abundance than geese from $\mathrm{CZ}$, suggesting that the species might suffer more various pathogens stress under harsh living conditions wintering at SJ. The SJ and CZ are crucial habitat for poultry, livestock areas, pathogens in the faeces of Anser erythropus could easily contaminate water, air and soil, in addition, the poultry often foraged together with wintering waterbirds in paddy fields at two lakes, and they could propagate the gut pathogens to each other and also increase the risk of diseases in other sympatric animals [17]. In addition, local residents have a lot of contact with wintering waterbirds and domestic poultry, thus those gut pathogens of Anser erythropus could propagate to human beings $[14,20]$. So, it is necessary to avoid the mixed group of wintering waterbirds and poultry to prevent the spread of pathogens to human society $[4,26]$.

In conclusion, environmental homogeneous and especially the differences in diets of the geese lead to the variations in gut fungal communities between Anser erythropus wintering at $\mathrm{SJ}$ and $\mathrm{CZ}$ in China. It also may be related to the fact that the Anser erythropus from SJ had migrated from a different breeding area than those from CZ (Liu et al. 2020). Gut potentially pathogenic communities were significantly different between two Anser erythropus samples as well as gut fungal communities. This study provides a foundation understanding of gut fungal community and potentially pathogene in Anser erythropus. There were certain limitations in this study, for example, only 20 replicates were selected for study, and 
spatial-temporal was not considered, these might be important factors to influence gut fungal community composition. These limitations should be clarified in future studies.

\section{Declarations}

\section{Funding Information}

This research was supported by the National Natural Science Foundation of China (grant no. 31702030).

\section{Data Availability}

The raw sequence data files were processed by NCBI SRA database and are accessible under the accession number SRR15328848.

\section{Compliance with Ethical Standards}

Conflict of Interest The authors declare that they have no conflict of interest.

\section{Ethics Approval and Consent to Participate}

No animals were harmed during this research. All experimental procedures complied with current laws regarding animal welfare and research in China and were specifically approved by the Animal Research Ethics Committee of Anhui Medical University.

Consent for Publication Not applicable.

\section{References}

[1] Ferlay J, Soerjomataram I, Dikshit R, Eser S, Mathers C, Rebelo M, Parkin DM, Forman D, Bray F (2015) Cancer incidence and mortality worldwide: sources, methods and major patterns in GLOBOCAN 2012. International Journal of Cancer 136(5):E359-86.

[2] Gao R, Gao Z, Huang L, Qin H (2017) Gut microbiota and colorectal cancer. European Journal of Clinical Microbiology and Infectious Diseases 36:757-769.

[3] Zhao Y, Li K, Luo HQ, Duan LC, Wei CX, Wang M, Jin JJ, Liu SZ, Mehmood K, Shahzad M (2019) Comparison of the intestinal microbial community in ducks reared differently through high-throughput sequencing. Biomed Research International 9015054.

[4] Fu R, Xiang XJ, Dong YQ, Cheng L, Zhou LZ (2020) Comparing the intestinal bacterial communies of sympatric wintering Hooded Crane (Grus monacha) and Domestic Goose (Anser anser domesticus). Avian Research 11:13. 
[5] Hammons S, Oh PL, Martínez I, Clark K, Schlegel VL, Sitorius E, Scheideler SE, Walter J (2010) A small variation in diet influences the Lactobacillus strain composition in the crop of broiler chickens.

Systematic and Applied Microbiology 33(5):275-81.

[6] Hird SM, Sánchez C, Carstens BC, Brumfield RT (2015) Comparative gut microbiota of 59 neotropical bird species. Frontiers in Microbiology 6: 1403.

[7] Barbosa A, Balagué V, Valera F, Martínez A, Benzal J, Motas M, Diaz Jl, Mira A, Pedrós-Alió C (2016) Age-related differences in the gastrointestinal microbiota of Chinstrap Penguins (Pygoscelis antarctica). PLOS ONE 11:0153215.

[8] Wang W, Zheng S, Sharshov K, Cao J, Sun H, Yang F, Wang X, Li L (2016) Distinctive gut microbial community structure in both the wild and farmed Swan goose (Anser cygnoides). Journal of Basic Microbiology 56:1299-1307.

[9] Wang W, Zheng S, Sharshov K, Sun H, Yang F, Wang X, Li L, Xiao Z (2017) Metagenomic profiling of gut microbial communities in both wild and artificially reared Barheaded goose (Anser indicus). Microbiologyopen 6:00429.

[10] Liu G, Gong ZZ, Li QY (2020) Variations in gut bacterial communities between lesser white-fronted geese wintering at Caizi and Shengjin lakes in China. Microbiologyopen 9(7):e1037.

[11] Palamidi I, Mountzouris KC (2018) Diet supplementation with an organic acidsbased formulation affects gut microbiota and expression of gut barrier genes in broilers. Animal Nutrition 4:367-77.

[12] Underhill DM, lliev ID (2014) The mycobiota: interactions between commensal fungi and the host immune system. Nature Reviews Immunology 14(6):405-16.

[13] Li JY, Chen DW, Yu B, He J, Huang ZQ, Mao XB, Zheng P, Yu J, Luo JQ, Tian G, Luo YH (2020) The fungal community and its interaction with the concentration of short-chain fatty acids in the faeces of Chenghua, Yorkshire and Tibetan pigs. Microbial Biotechnology 13(2):509-521.

[14] Xiang XJ, Jin LL, Yang ZQ, Zhang NZ, Zhang F (2021) Dramatic shifts in intestinal fungal community between wintering Hooded Crane and Domestic Goose. Avian Research 12:1.

[15] Allali I, Arnold JW, Roach J, Cadenas MB, Butz N, Hassan HM, Koci M, Ballou A, Mendoza M, Ali R, Azcarate-Peril MAA (2017) Comparison of sequencing platforms and bioinformatics pipelines for compositional analysis of the gut microbiome. BMC Microbiology 17: 194.

[16]Wang W, Wang AZ, Yang YS, Wang F, Liu YB, Zhang YH, Sharshov K, Gui LS (2019) Composition, diversity and function of gastrointestinal microbiota in wild red-billed choughs (Pyrrhocorax pyrrhocorax), International Journal of Microbiology 22(4):491-500. 
[17] Yang YZ, Deng Y, Cao L (2016) Characterising the interspecific variations and convergence of gut microbiota in Anseriformes herbivores at wintering areas. Scientific Reports 6: 32655.

[18] Chen JY, Zhou LZ, Zhou B, Xu RX, Zhu WZ, Xu WB (2011) Seasonal dynamics of wintering waterbirds in two shallow lakes along Yangtze River in Anhui Province. Zoological Research 32: 540-548.

[19] Dong YQ, Xiang XJ, Zhao GH, Song YW, Zhou LZ (2019) Variations in gut bacterial communities of hooded crane (Grus monacha) over spatial-temporal scales. PeerJ 7:e7045.

[20] Xiang XJ, Zhang FL, Fu R, Yan SF, Zhou LZ (2019) Significant differences in bacterial and potentially pathogenic communities between sympatric Hooded crane and greater white-fronted goose. Frontiers in Microbiology 10:163.

[21] Lara BR, Milena Melo B de A, Paula CR, Arnoni MV, Simões CCN, Nakano S, Richini-Pereira VB, Garces HG, Maciel da Silva BC, Anversa L, Silva EG, Auler ME, Oliveira Dos Santos RL, da Silva Ruiz L (2019) Apiotrichum veenhuisii isolated from a pediatric patient with acute myeloid leukemia: the first case in humans. Mycologia 111(5):793-797.

[22] Li J, Chen D, Yu B, He J, Zheng P, Mao X, Yu J, Luo J, Tian G, Huang Z, Luo Y (2017) Fungi in gastrointestinal tracts of human and mice: from community to functions. Microbial Ecology 75: 821829.

[22] Zhao MJ, Cong PH, Barter M, Fox AD, Cao L (2012) The changing abundance and distribution of greater white-fronted geese Anser albifrons in the Yangtze River floodplain: impacts of recent hydrological changes. Bird Conservation International 22(2): 135-143.

[23] Bolnick DI, Snowberg LK, Hirsch PE, Lauber CL, Org E, Parks B, Lusis AJ, Knight R, Caporaso JG, Svanbäck R (2014) Individual diet has sex-dependent effects on vertebrate gut microbiota. Nature Communications 29, (5):4500.

[24] Wang X, Fox AD, Cong P, Barter M, Cao L (2012) Changes in the distribution and abundance of wintering Lesser White-fronted Geese Anser erythropus in eastern China. Bird Conservation International 22(2): 128.

[25] Zhao GH, Zhou LZ, Dong YQ, Cheng YY, Song YW (2017) The gut microbiome of hooded cranes (Grus monacha) wintering at Shengjin Lake, China. Microbiologyopen 6(3):e00447.

[26] Kevin DK (2012) Diversity and function of the avian gut microbiota. Journal of Comparative Physiology B-Biochemical Systems and Environmental Physiology 182:591-602.

\section{Figures}



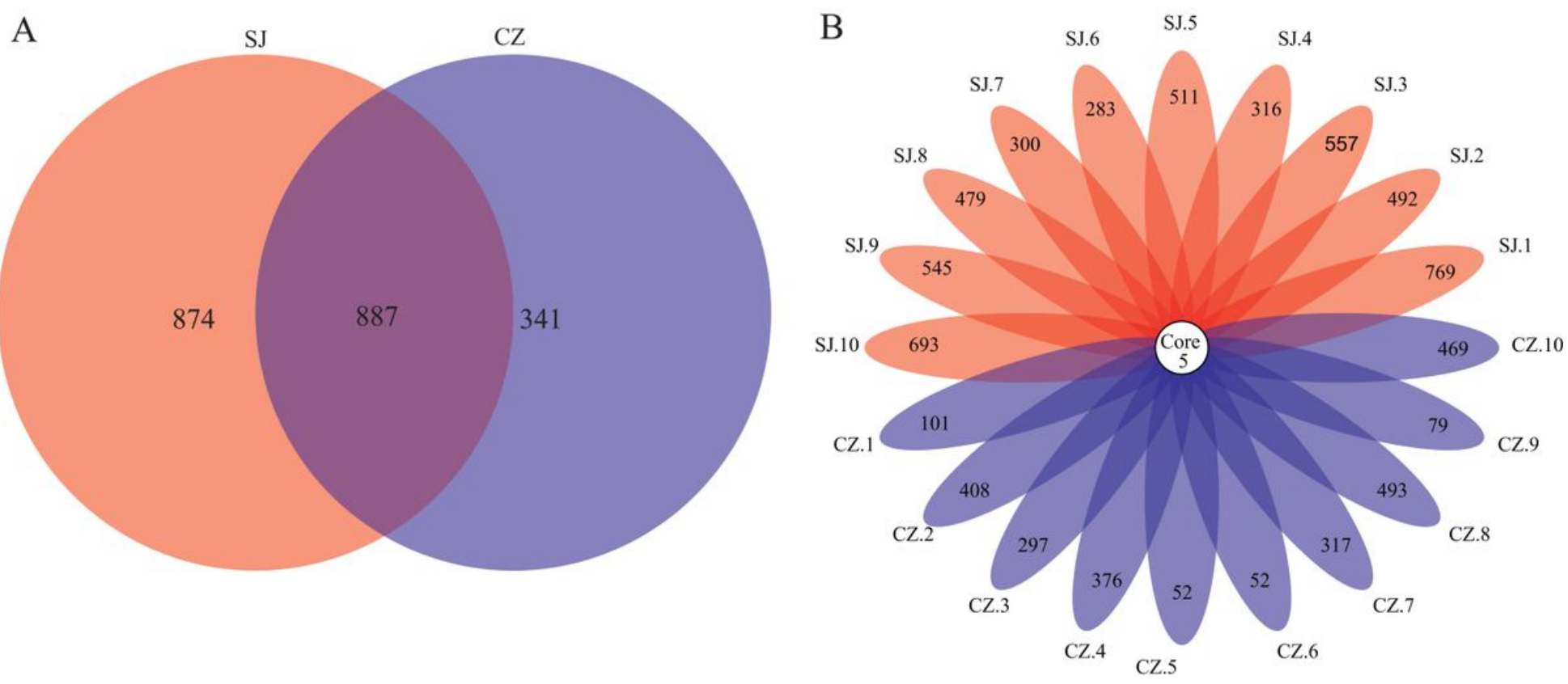

\section{Figure 1}

Venn diagrams illustrating the number of OTUs (A) and core-OTUs (B) for gut fungi of Anser erythropus from SJ and CZ. 
A

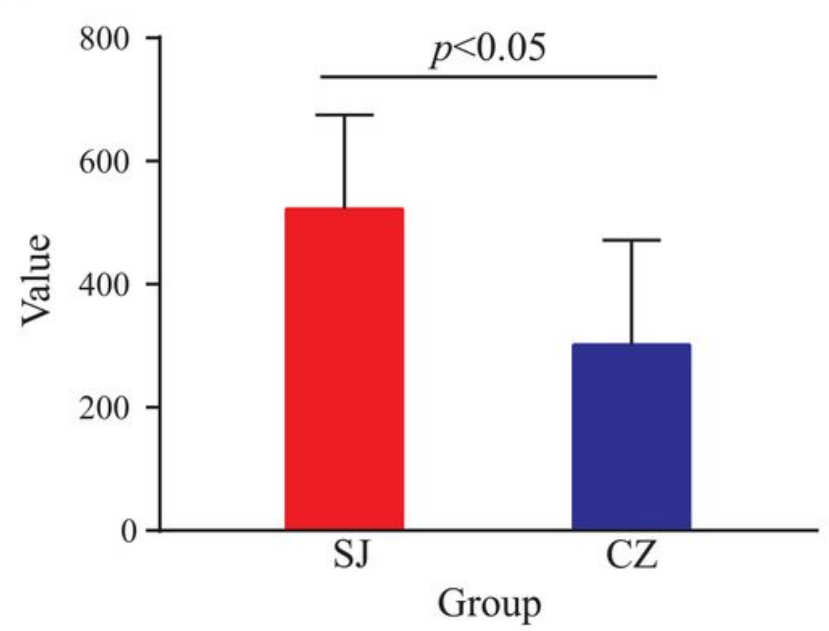

B

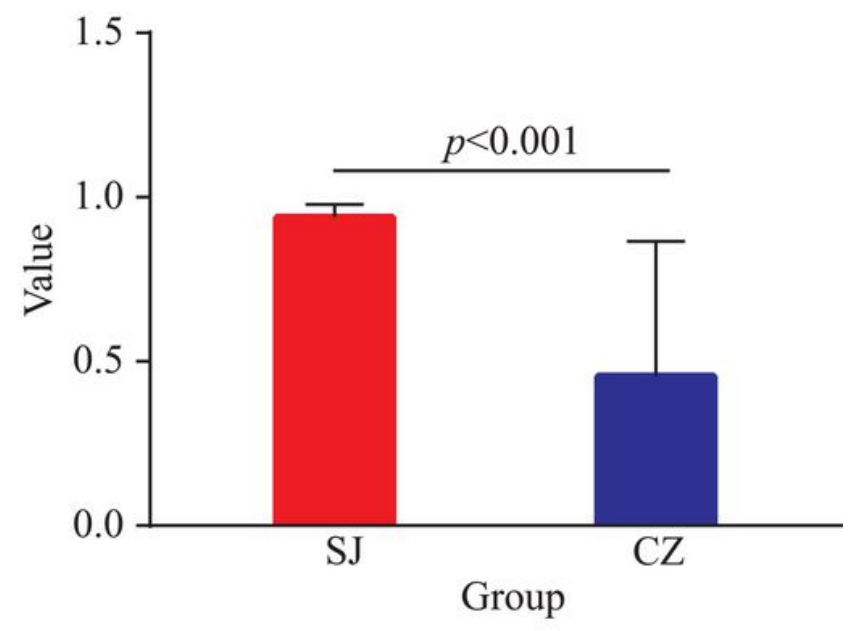

$\mathrm{C}$

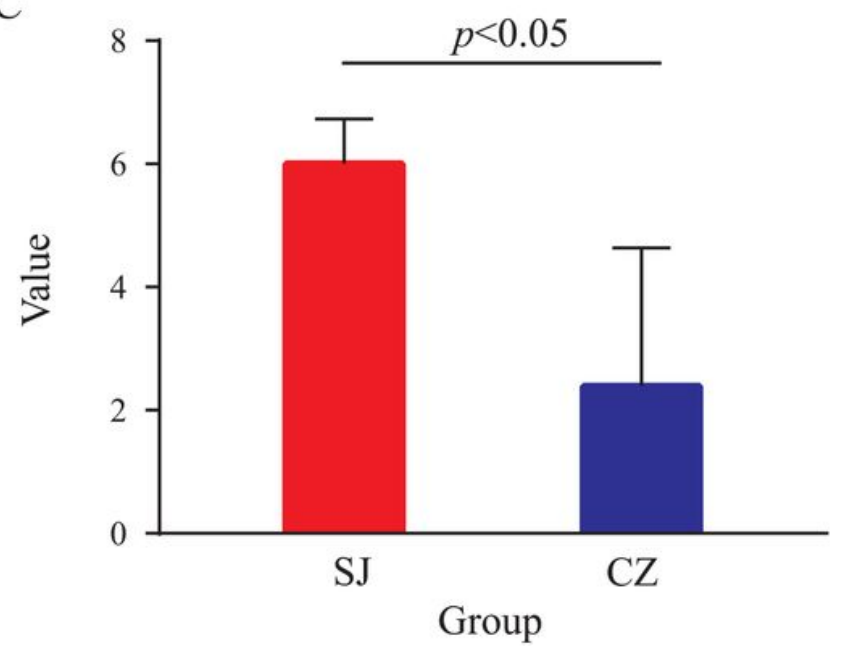

Figure 2

Chao1 (A), Simpson (B), and Shannon (C) indices for the gut fungi from Anser erythropus at SJ and CZ. 


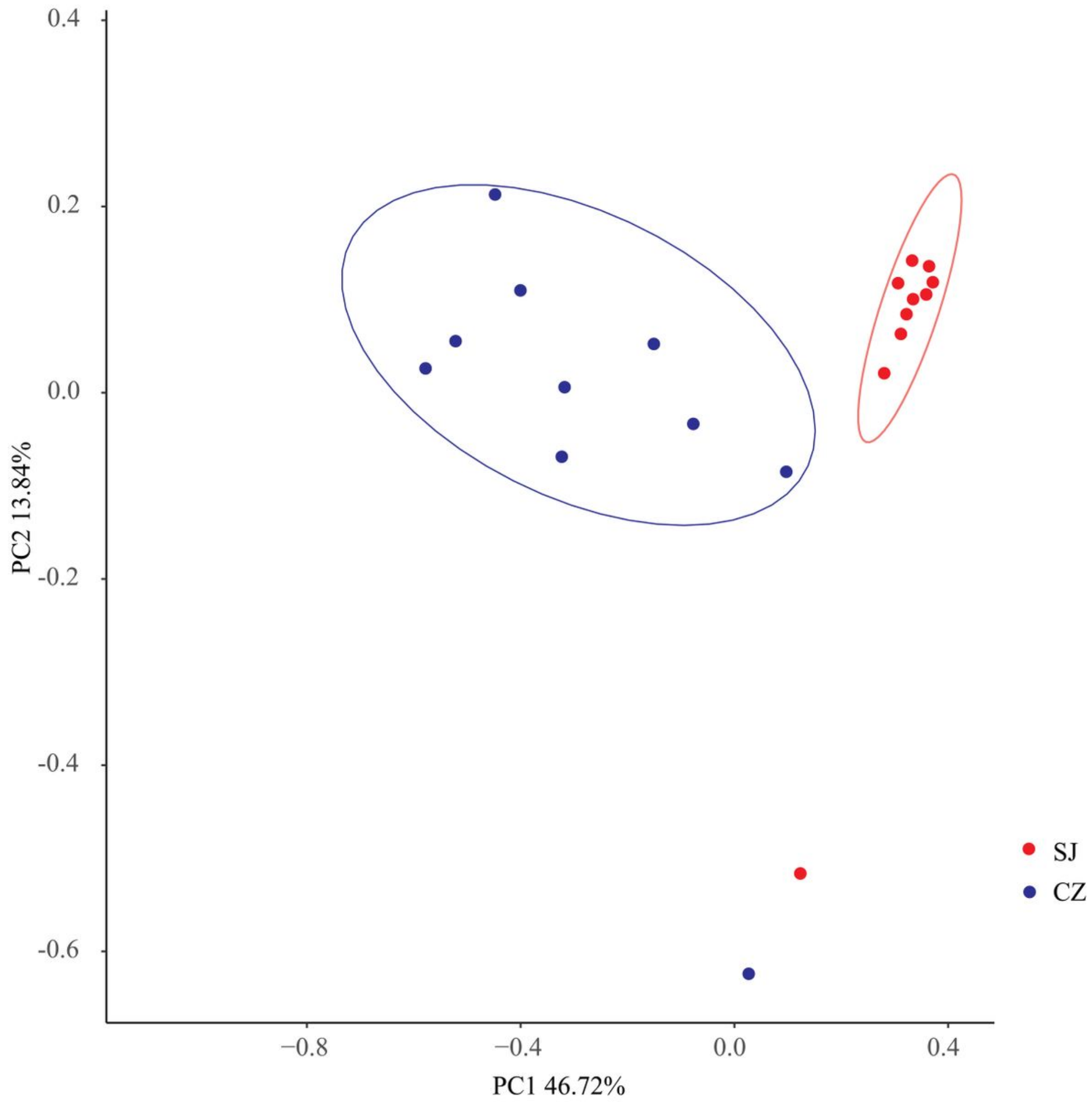

Figure 3

PCoA analyze result in fecal gut for the Anser erythropus sampled from SJand CZ. Red: fecal samples of geese from SJ; blue: fecal samples of geese from CZ. 


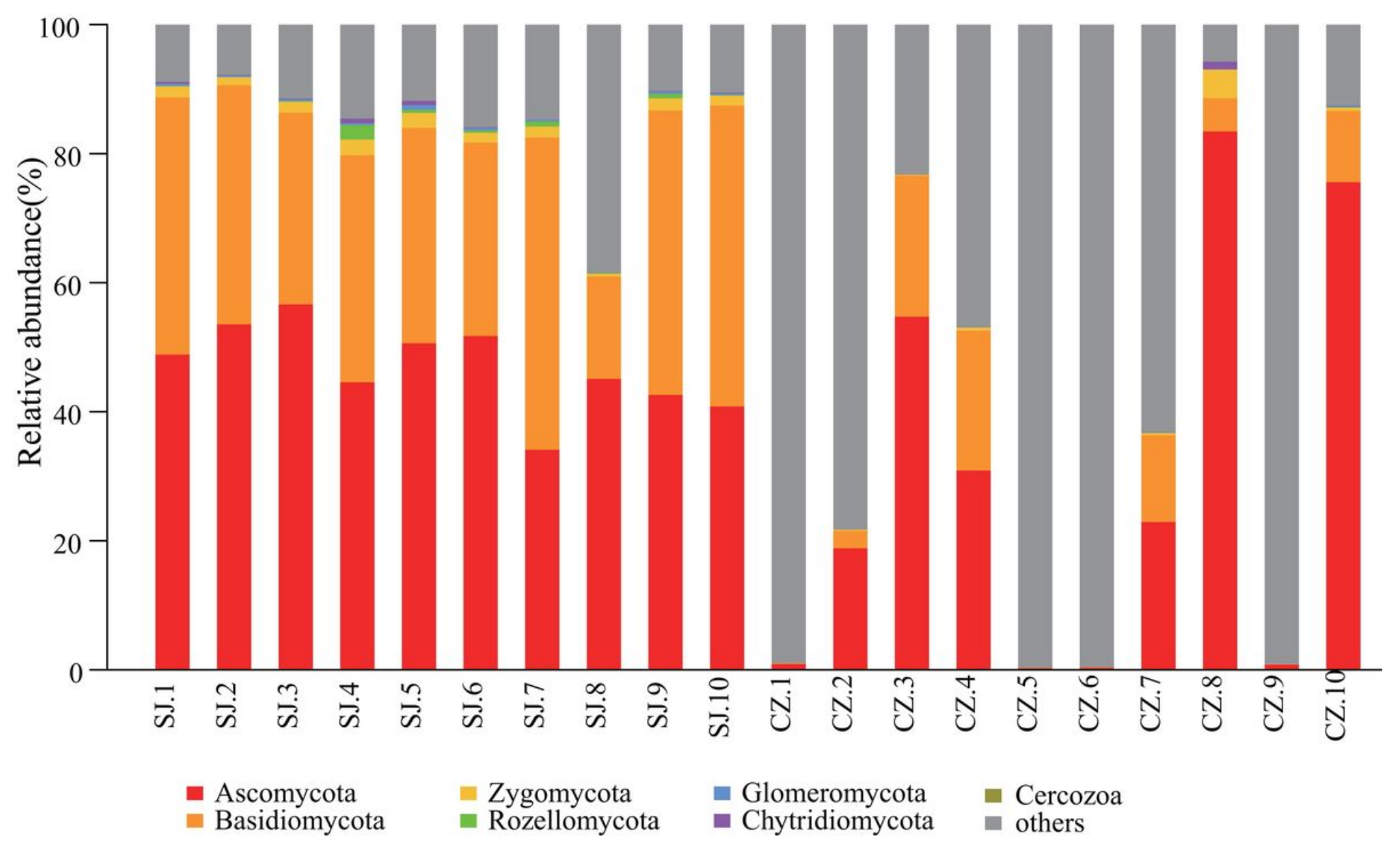

Figure 4

The dominant gut fungal phyla in the Anser erythropus at SJ and CZ. 


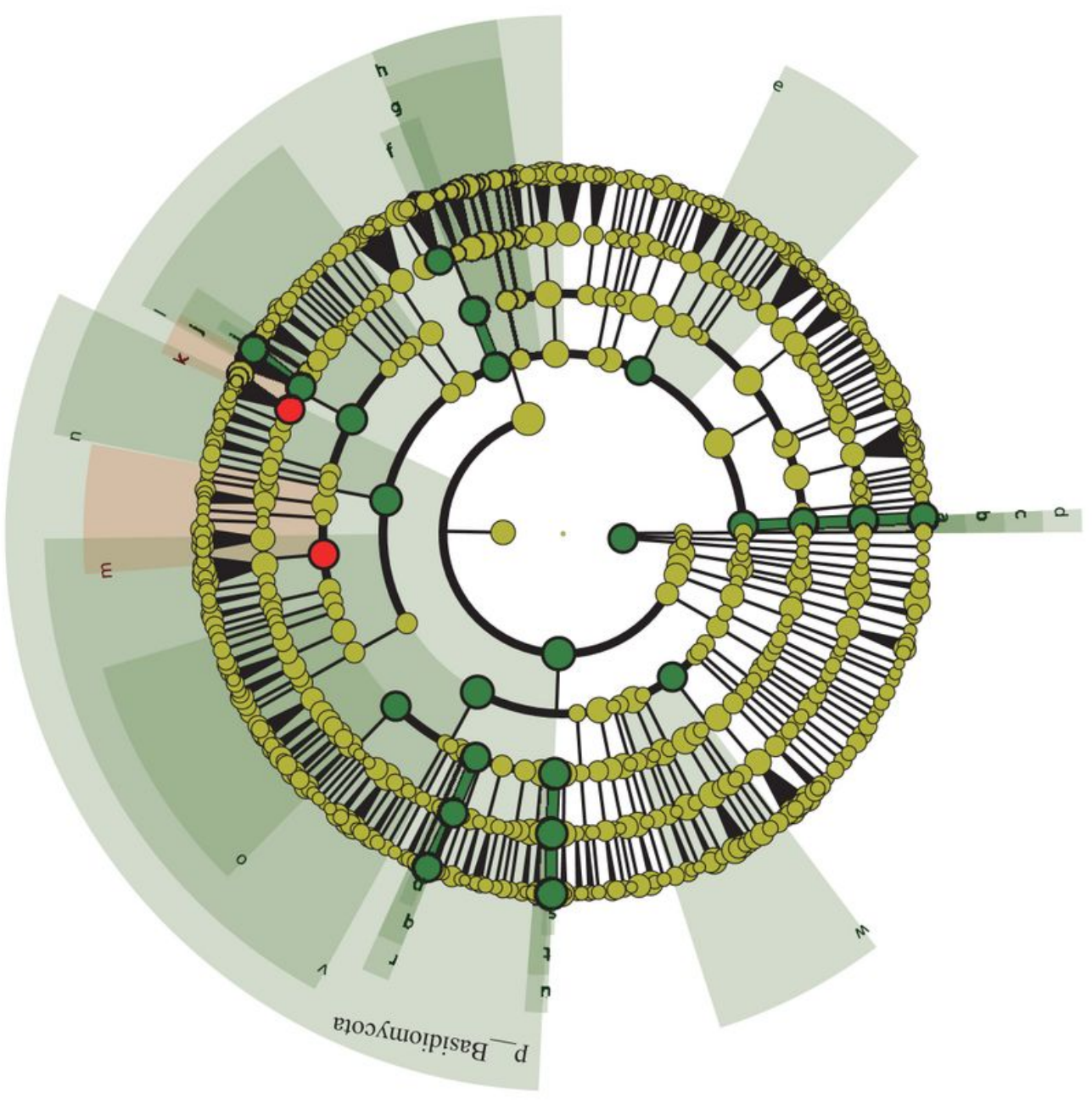

$\square$ a: g_Archaeorhizomyces

b: f_Archaeorhizomycetaceae

c: o Archaeorhizomycetales

$\square \mathrm{d}$ : c__Archaeorhizomycetes

e: c Eurotiomycetes

$\square \mathrm{f}$ : f_Pyronemataceae

g: o__Pezizales

h: c_Pezizomycetes

i: g_Acremonium

j: $f$ Hypocreales fam Incertae sedis

k: f_Nectriaceae

1:0_Hypocreales

m: o_Sordariales

n: c_Sordariomycetes

o: o_Agaricales

p: g_Ceratobasidium

q: f_Ceratobasidiaceae

r: o_Cantharellales

s: g_ Tomentella

t: f_Thelephoraceae

u: o Thelephorales

v: c Agaricomycetes

w: c_Tremellomycetes

\section{Figure 5}

LEfSe analysis of the gut fungi from Anser erythropus at SJ and CZ. 


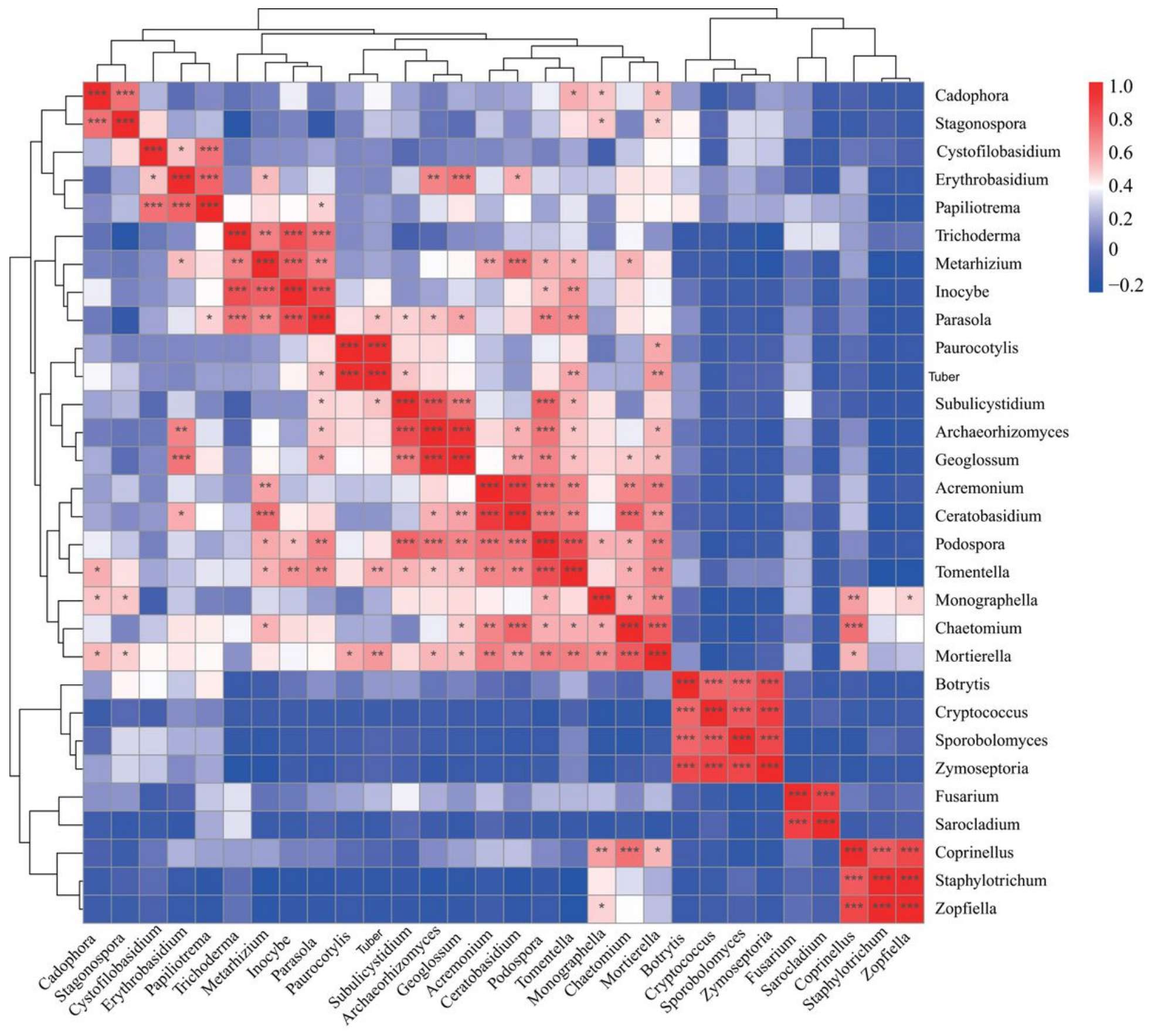

Figure 6

Cor-Correlation among the top 30 core genera across the 20 samples from Anser erythropus at SJ and $C Z$, as determined by the Spearman's rank correlation analysis. Notes: ${ }^{*} p<0.05,{ }^{* *} p<0.01$ and ${ }^{* \star *} p$ $<0.001$. Red is positively correlation and blue is negatively correlation. 


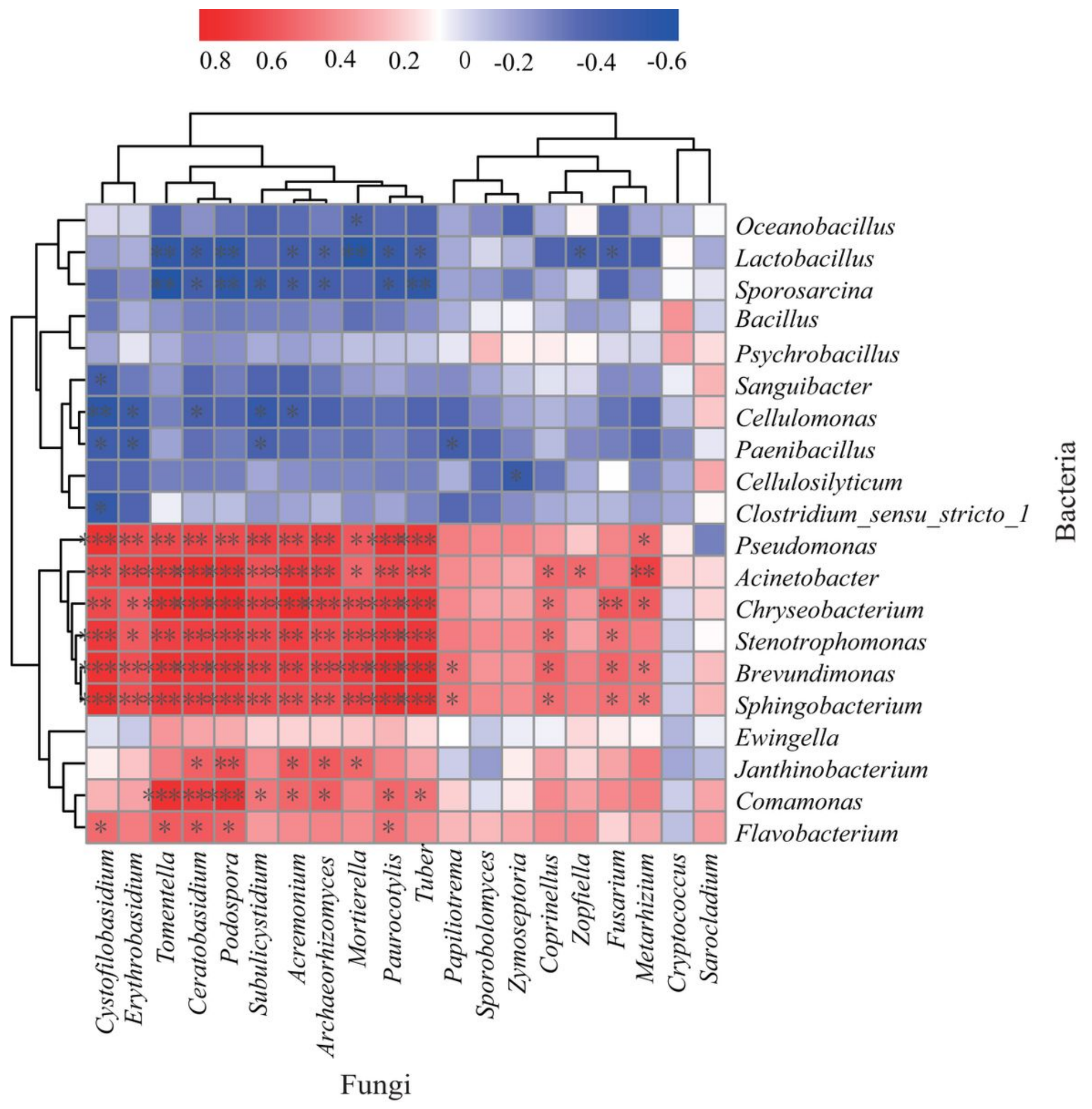

Figure 7

Specific bacteria-fungi correlation analysis result in the 20 samples from Anser erythropus at SJ and CZ. Notes: ${ }^{*} p<0.05,{ }^{* *} p<0.01$ and ${ }^{* \star *} p<0.001$. Red is positively correlation and blue is negatively correlation. 


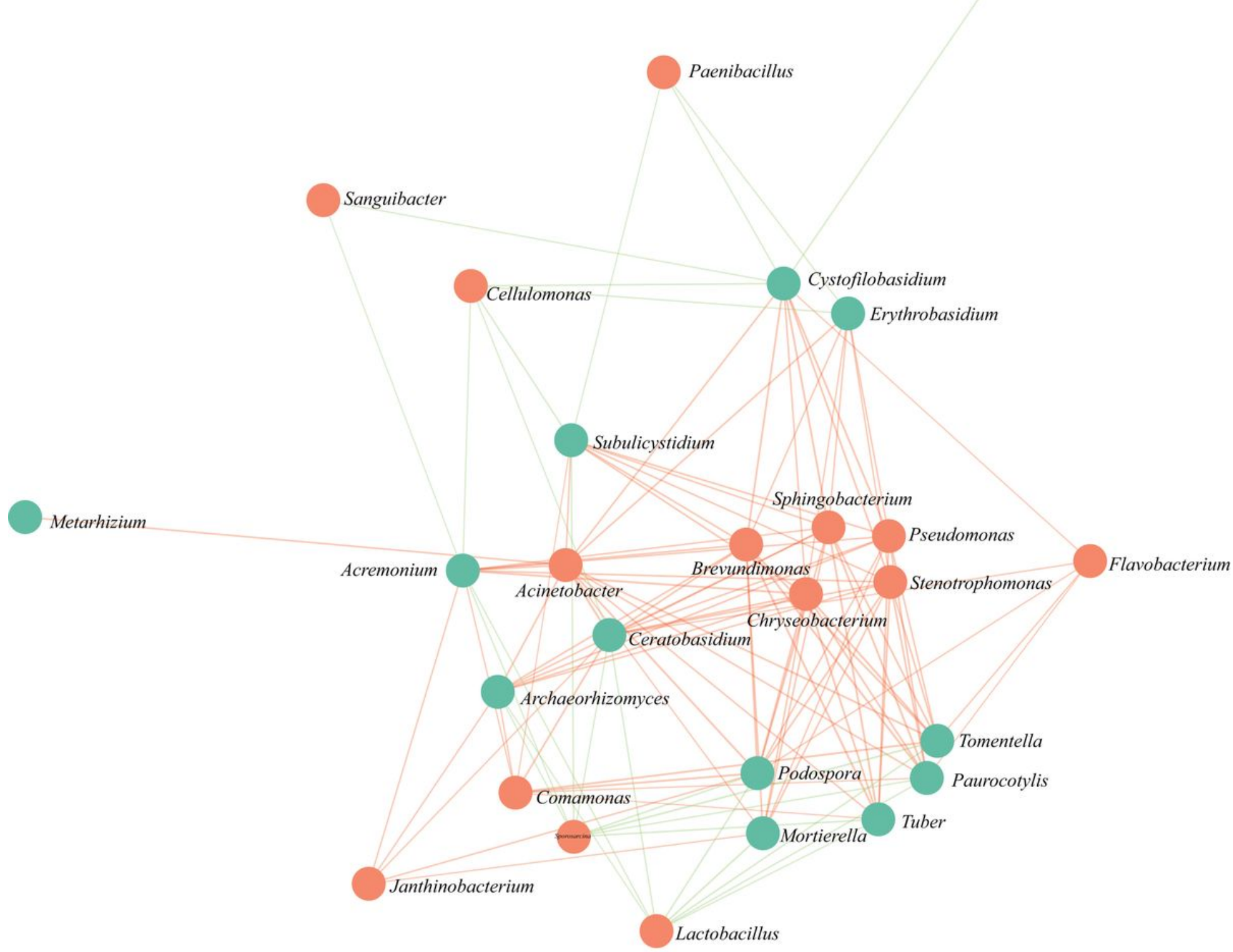

\section{Fungi Bacteria}

\section{Figure 8}

Correlation network between fungi and bacteria from Anser erythropus at SJ and CZ. Orange circles indicate positive correlations, and green circles indicate inverse correlations. 
A

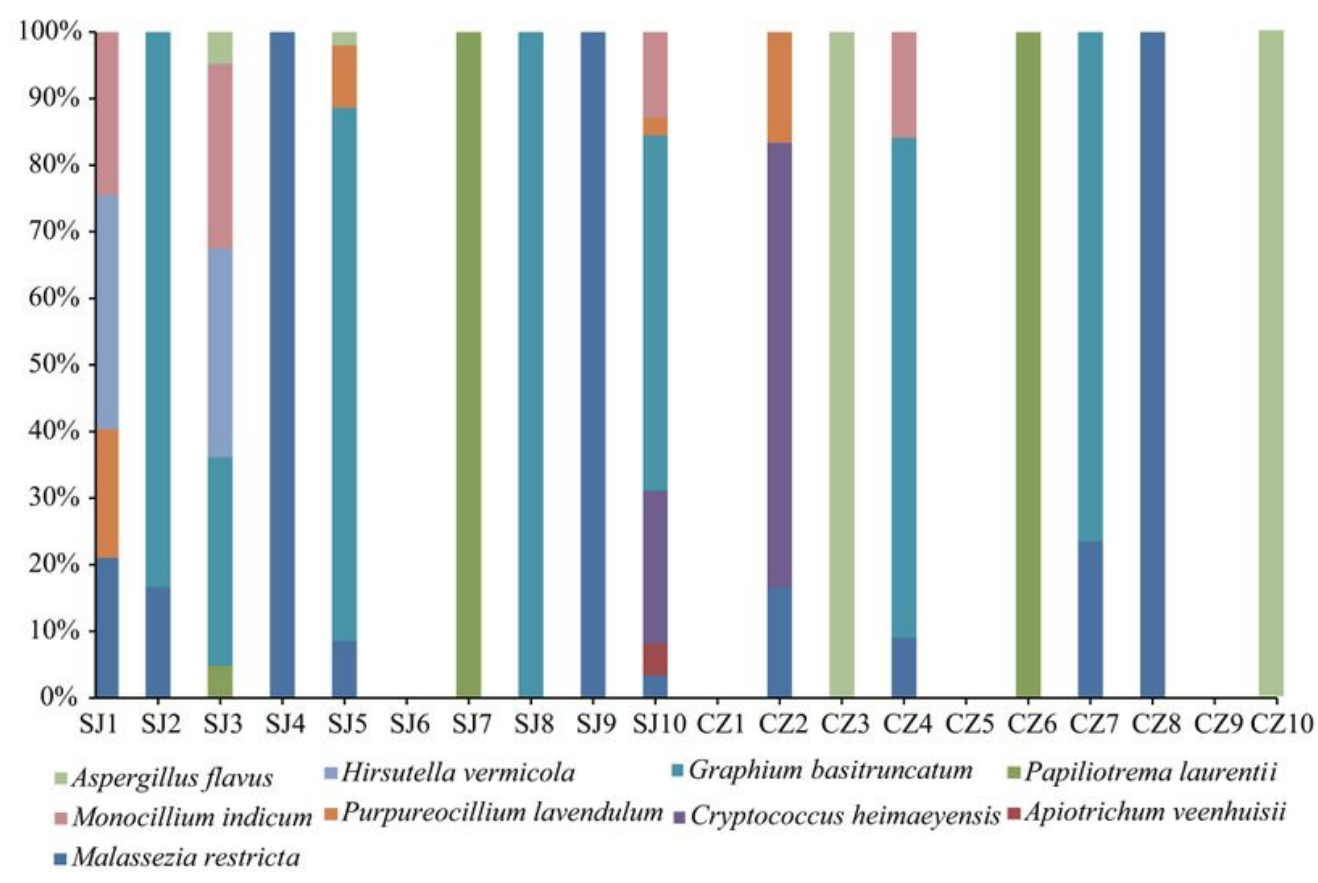

B

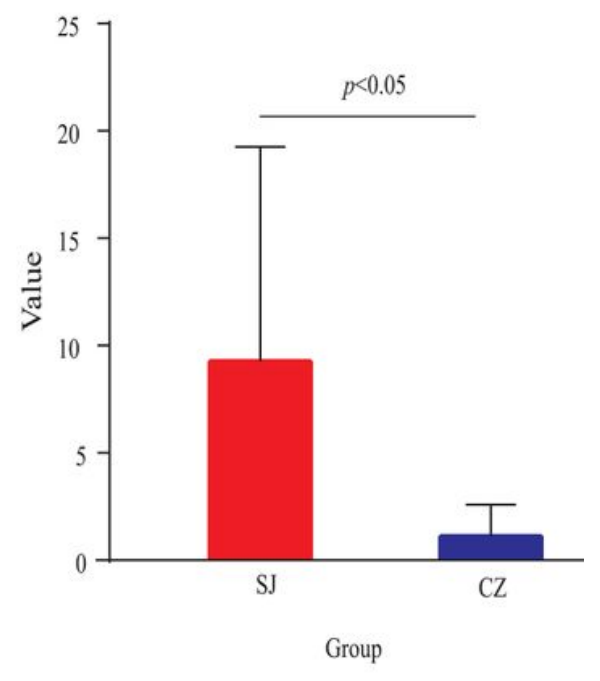

Figure 9

Composition of potential pathogenic species (A) and animal pathogenic diversity (B) of the gut fungi from Anser erythropus at SJ and CZ. 